\title{
Nonlinear Dynamic Control and Friction Compensation of Parallel Manipulators
}

\author{
Weiwei Shang and Shuang Cong \\ University of Science and Technology of China \\ P.R. China
}

\section{Introduction}

Comparing with the serial ones, parallel manipulators have potential advantages in terms of high stiffness, accuracy and speed (Merlet, 2001). Especially the high accuracy and speed performances make the parallel manipulators widely applied to the following fields, like the pick-and-place operation in food, medicine, electronic industry and so on. At present, the key issues are the ways to meet the demand of high accuracy in moving process under the condition of high speed. In order to realize the high speed and accuracy motion, it's very important to design efficient control strategies for parallel manipulators.

In literatures, there are two basic control strategies for parallel manipulators (Zhang et.al., 2007): kinematic control strategies and dynamic control strategies. In the kinematic control strategies, parallel manipulators are decoupled into a group of single axis control systems, so they can be controlled by a group of individual controllers. Proportional-derivative (PD) control(Ghorbel et.al., 2000; Wu et.al., 2002), nonlinear PD (NPD) control (Ouyang et.al., 2002; Su et.al., 2004), and fuzzy control (Su et.al., 2005) all belong to this type of control strategies. These controllers do not always produce high control performance, and there is no guarantee of stability at the high speed. Unlike the kinematic control strategies, full dynamic model of parallel manipulators is taken into account in the dynamic control strategies. So the nonlinear dynamics of parallel manipulators can be compensated and better performance can be achieved with the dynamic strategies.

The traditional dynamic control strategies of parallel manipulators are the augmented PD (APD) control and the computed-torque (CT) control (Li \& Wu, 2004; Cheng et.al., 2003; Paccot et.al., 2009). In the APD controller (Cheng et.al., 2003), the control law contains the tracking control term and the feed-forward compensation term. The tracking control term is realized by the PD control algorithm. The feed-forward compensation term contains the dynamic compensation calculated by the desired velocity and desired acceleration on the basis of the dynamic model. Compared with the simple PD controller, the APD controller is a tracking control method. However, the feed-forward compensation can not restrain the trajectory disturbance effectively, thus the tracking accuracy of the APD controller will be decreased. In order to solve this problem, the CT controller including the velocity feed-back is proposed based on the PD controller (Paccot et.al., 2009). The CT control method yields a controller that suppresses disturbance and tracks desired trajectories uniformly in all configurations of the manipulators. Both the APD controller and the CT controller contain two parts including the PD control term and the dynamic compensation term. For the 
presence of nonlinear factors such as modeling error and nonlinear friction in the dynamic models of the parallel manipulators, those traditional controllers can not achieve good control accuracy.

In order to overcome the uncertain factors in parallel manipulators, nonlinear control methods and friction compensation method are developed in this chapter. Firstly, in order to restrain the modeling error of parallel manipulators, a nonlinear PD (NPD) control algorithm is used to the APD controller, and a so-called augmented NPD (ANPD) controller is designed. Secondly, considering the feed-forward compensation term in the ANPD controller can not restrict the external disturbance, and the tracking accuracy will be affected when the disturbance exists. Thus the NPD controller is combined with the CT controller further, and a new control method named nonlinear CT (NCT) controller is developed. Thirdly, in order to compensate the nonlinear friction of parallel manipulators, a nonlinear model with two-sigmoid-function is introduced to modeling the nonlinear friction. This nonlinear friction model enables reconstruction of viscous, Coulomb, and Stribeck friction effects of parallel manipulators, and the nonlinear optimization tool is used to estimate the parameters in this model. In addition to the theoretical development, all the proposed methods in this chapter are validated on an actual parallel manipulator. The experiment results indicate that, compared with the conventional controllers, the proposed ANPD and NCT controller can get better trajectory tracking accuracy of the end-effector. Moreover, the experiment results also demonstrate that the nonlinear friction model is more accurately to compensate the friction, and is robust against the trajectory and the velocity changes.

\section{Dynamic modelling}

The experiment platform is a 2-DOF parallel manipulator with redundant actuation. As shown in Fig. 1, a reference frame is established in the workspace of the parallel manipulator. The unit of the frame is meter. The parallel manipulator is actuated by three servo motors located at the base A1, A2, and A3, and the end-effector is mounted at the common joint $\mathrm{O}$, where the three chains meet. Coordinates of the three bases are $\mathrm{A} 1(0,0.25)$, A2 $(0.433,0)$, and A3 $(0.433,0.5)$, and all of the links have the same length $l=0.244 \mathrm{~m}$. The definitions of the joint angles are shown in the Fig. $1, q_{a 1}, q_{a 2}, q_{a 3}$ refer to the active joint angles and $q_{b 1}, q_{b 2}, q_{b 3}$ refer to the passive joint angles.

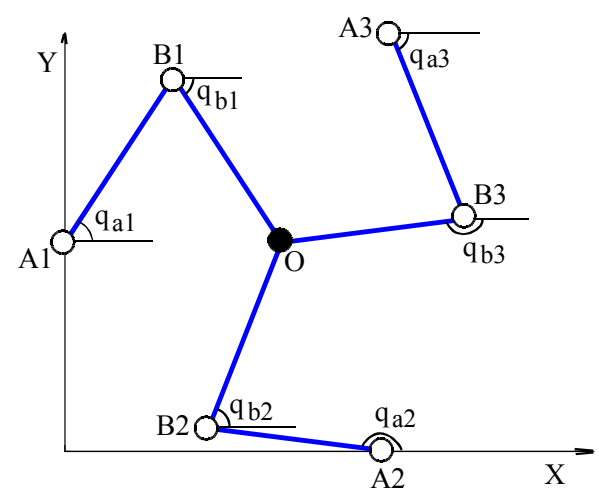

Fig. 1. Coordinates of the 2-DOF parallel manipulator with redundant actuation 
Cutting the parallel manipulator at the common point $O$ in Fig. 1, one can have an openchain system including three independent planar 2-DOF serial manipulators, each of which contains an active joint and a passive joint. The dynamic model of the parallel manipulator equals to the model of the open-chain system plus the closed-loop constraints, thus the dynamic model of the whole parallel manipulator can be formulated by combining the dynamics of the three serial manipulators under the constraints.

As we know, the dynamic model of each planar 2-DOF serial manipulator can be formulated as (Murray et.al., 1994)

$$
\mathbf{M}_{i} \ddot{\mathbf{q}}_{i}+\mathbf{C}_{i} \dot{\mathbf{q}}_{i}+\mathbf{f}_{i}=\mathbf{\tau}_{i}
$$

where $\mathbf{q}_{i}=\left[\begin{array}{ll}q_{a i} & q_{b i}\end{array}\right]^{T}, q_{a i}$ and $q_{b i}$ are the active joint and passive joint angle, respectively; $\mathbf{M}_{i}$ is inertia matrix, and $\mathbf{C}_{i}$ is Coriolis and centrifugal force matrix, which are defined as

$$
\begin{gathered}
\mathbf{M}_{i}=\left[\begin{array}{cc}
\alpha_{i} & \gamma_{i} \cos \left(q_{a i}-q_{b i}\right) \\
\gamma_{i} \cos \left(q_{a i}-q_{b i}\right) & \beta_{i}
\end{array}\right] \\
\mathbf{C}_{i}=\left[\begin{array}{cc}
0 & \gamma_{i} \sin \left(q_{a i}-q_{b i}\right) \dot{q}_{b i} \\
-\gamma_{i} \sin \left(q_{a i}-q_{b i}\right) \dot{q}_{a i} & 0
\end{array}\right]
\end{gathered}
$$

where $\alpha_{i}, \beta_{i}, \gamma_{i}, i=1,2,3$ are the dynamic parameters which are related with the physical parameters such as mass, center of mass, and inertia. In Eq.(1), $\mathbf{\tau}_{i}=\left[\begin{array}{ll}\tau_{a i} & \tau_{b i}\end{array}\right]^{T}$ is joint torque vector, where $\tau_{a i}$ is the active joint torque, the passive joint torque $\tau_{b i}=0$. Vector $\mathbf{f}_{i}=\left[\begin{array}{ll}f_{a i} & f_{b i}\end{array}\right]^{T}$ is the friction torque, where $f_{a i}$ and $f_{b i}$ are the active joint friction and passive joint friction, respectively. The friction parameters of the active joints and the passive joints are identified simultaneously for the parallel manipulator (Shang et.al., 2010). And from the identified results, one can find that the friction parameters of the passive joints are much smaller than those of the active joints. Thus, compared with the active joints friction $f_{a i}$, the passive joint friction $f_{b i}$ is much smaller and it can be neglected (Shang et.al., 2010). Generally, the active joint friction torque $f_{a i}$ can be formulated by using the Coulomb + viscous friction model as

$$
f_{a i}=\operatorname{sign}\left(\dot{q}_{a i}\right) f_{c i}+f_{v i} \dot{q}_{a i}
$$

where $f_{c i}$ represents the Coulomb friction, and $f_{v i}$ represents the coefficient of the viscous friction.

Combining the dynamic models of three 2-DOF serial manipulators, the dynamic model of the open-chain system can be expressed as

$$
\mathbf{M} \ddot{\mathbf{q}}+\mathbf{C} \dot{\mathbf{q}}+\mathbf{f}=\mathbf{\tau}
$$

where the definition of the symbols is similar to those in Eq.(1), only the difference is that the symbols in Eq.(3) represent the whole open-chain system not a 2-DOF serial manipulator. Based on Eq.(3) of the open-chain system and the constraint forces due to the closed-loop constraints, the dynamic model of the parallel manipulator can be written as

$$
\mathbf{M} \ddot{\mathbf{q}}+\mathbf{C} \dot{\mathbf{q}}+\mathbf{f}=\mathbf{\tau}+\mathbf{A}^{T} \boldsymbol{\lambda}
$$


where $\mathbf{A}^{T} \boldsymbol{\lambda}$ represents the constraint force vector, here matrix $\mathbf{A}$ is the differential of the closed-loop constrained equation and $\lambda$ is a unknown multiplier representing the magnitude of the constraint forces. Fortunately, $\mathbf{A}^{T} \boldsymbol{\lambda}$ can be eliminated, by finding the nullspace of matrix $\mathbf{A}$ (Muller, 2005). With the Jacobian matrix $\mathbf{W}$, we have

$$
\dot{\mathbf{q}}=\mathbf{W} \dot{\mathbf{q}}_{e}
$$

where $\quad \dot{\mathbf{q}}=\left[\begin{array}{llllll}\dot{q}_{q_{1} 1} & \dot{q}_{a 2} & \dot{q}_{a 3} & \dot{q}_{b 1} & \dot{q}_{b 2} & \dot{q}_{b 3}\end{array}\right]^{T}$ represents the velocity vector of all the joints, $\dot{\mathbf{q}}_{e}=\left[\begin{array}{ll}\dot{q}_{x} & \dot{q}_{y}\end{array}\right]^{1}$ represents the velocity vector of the end-effector, and the Jacobian matrix $\mathbf{W}$ is defined as

$$
\mathbf{W}=\left[\begin{array}{cc}
r_{1} \cos \left(q_{b 1}\right) & r_{1} \sin \left(q_{b 1}\right) \\
r_{2} \cos \left(q_{b 2}\right) & r_{2} \sin \left(q_{b 2}\right) \\
r_{3} \cos \left(q_{b 3}\right) & r_{3} \sin \left(q_{b 3}\right) \\
-r_{1} \cos \left(q_{a 1}\right) & -r_{1} \sin \left(q_{a 1}\right) \\
-r_{2} \cos \left(q_{a 2}\right) & -r_{2} \sin \left(q_{a 2}\right) \\
-r_{3} \cos \left(q_{a 3}\right) & -r_{3} \sin \left(q_{a 3}\right)
\end{array}\right] \text {, where } r_{i}=\frac{1}{l \sin \left(q_{b i}-q_{a i}\right)}
$$

Considering the constraint equation $\mathbf{A} \dot{\mathbf{q}}=\mathbf{0}$, then one can have $\mathbf{A W} \dot{\mathbf{q}}_{\mathbf{e}}=\mathbf{0}$ with the Jacobian relation Eq.(5). The velocity vector $\dot{\mathbf{q}}_{e}$ of the end-effector contains independent generalized coordinates, so one can get $\mathbf{A W}=\mathbf{0}$, or equivalently, $\mathbf{W}^{T} \mathbf{A}^{T}=\mathbf{0}$. With this result, the term of $\mathbf{A}^{T} \boldsymbol{\lambda}$ can be eliminated, and the dynamic model Eq. (4) can be written as

$$
\mathbf{W}^{T} \mathbf{M} \ddot{\mathbf{q}}+\mathbf{W}^{T} \mathbf{C} \dot{\mathbf{q}}+\mathbf{W}^{T} \mathbf{f}=\mathbf{W}^{T} \mathbf{\tau}+\mathbf{W}^{T} \mathbf{A}^{T} \boldsymbol{\lambda}=\mathbf{W}^{T} \mathbf{\tau}
$$

In order to study dynamic control and trajectory planning of the parallel manipulator both in the task space, we will further formulate the dynamic model in the task space on the basis of the dynamic model Eq. (6) of the joint space. Differentiating the Jacobian Eq. (5) yields

$$
\ddot{\mathbf{q}}=\dot{\mathbf{W}} \dot{\mathbf{q}}_{e}+\mathbf{W} \ddot{\mathbf{q}}_{e}
$$

and substituting Eqs. (5) and (7) into Eq. (6), the dynamic model in the task space can be written as

$$
\mathbf{W}^{T} \mathbf{M W} \ddot{\mathbf{q}}_{e}+\mathbf{W}^{T}(\mathbf{M} \dot{\mathbf{W}}+\mathbf{C W}) \dot{\mathbf{q}}_{e}+\mathbf{W}^{T} \mathbf{f}=\mathbf{W}^{T} \mathbf{\tau}
$$

If the friction torques of the passive joints is neglected, then Eq. (8) can be further simplified. Let $\boldsymbol{\tau}_{a}$ and $\mathbf{f}_{a}$ be the actuator and friction torque vector of the three active joints respectively, then $\mathbf{W}^{T} \mathbf{\tau}=\mathbf{S}^{T} \boldsymbol{\tau}_{a}$, and $\mathbf{W}^{T} \mathbf{f}=\mathbf{S}^{T} \mathbf{f}_{a}$. Here, $\mathbf{S}$ is the Jacobian matrix between the velocity of the end-effector and the velocity of three active joints, and $\mathbf{S}$ is written as

$$
\mathbf{S}=\left[\begin{array}{ll}
r_{1} \cos \left(q_{b 1}\right) & r_{1} \sin \left(q_{b 1}\right) \\
r_{2} \cos \left(q_{b 2}\right) & r_{2} \sin \left(q_{b 2}\right) \\
r_{3} \cos \left(q_{b 3}\right) & r_{3} \sin \left(q_{b 3}\right)
\end{array}\right]
$$

Then, the dynamic model in the task space can be written as 


$$
\mathbf{W}^{T} \mathbf{M W} \ddot{\mathbf{q}}_{e}+\mathbf{W}^{T}(\mathbf{M} \dot{\mathbf{W}}+\mathbf{C W}) \dot{\mathbf{q}}_{e}+\mathbf{S}^{T} \mathbf{f}_{a}=\mathbf{S}^{T} \mathbf{\tau}_{a}
$$

The above Eq.(9) can be briefly expressed as

$$
\mathbf{M}_{e} \ddot{\mathbf{q}}_{e}+\mathbf{C}_{e} \dot{\mathbf{q}}_{e}+\mathbf{S}^{T} \mathbf{f}_{a}=\mathbf{S}^{T} \mathbf{\tau}_{a}
$$

where $\mathbf{M}_{e}=\mathbf{W}^{T} \mathbf{M W}$ is the inertial matrix in the task space, and $\mathbf{C}_{e}=\mathbf{W}^{T}(\mathbf{M} \dot{\mathbf{W}}+\mathbf{C W})$ is the Coriolis and centrifugal force matrix in the task space.

The dynamic model Eq. (10) in the task space also satisfies the similar structural properties to the dynamic model of the open-chain system and the 2-DOF serial manipulator as follows (Cheng et.al., 2003):

a. $\quad \mathbf{M}_{e}$ is symmetric and positive.

b. $\quad \dot{\mathbf{M}}_{e}-\mathbf{2} \mathrm{C}_{e}$ is skew-symmetric matrix.

\section{Nonlinear dynamic control by using the NPD}

There are two conventional dynamic controllers for parallel manipulators: APD controller and CT controller. The common feature of the two controllers is eliminating the tracking error by linear PD control. However, the linear PD control is not robust against the uncertain factors such as modeling error and external disturbance. To overcome this problem, the NPD control can be combined with the conventional control strategies to improve the control accuracy and disturbance rejection ability.

\subsection{NPD controller}

As well as we know, the linear PD controller takes the form

$$
u_{L}(t)=k_{p} e(t)+k_{d} \dot{e}(t)
$$

where $k_{p}$ and $k_{d}$ are the proportional and derivative constants respectively, and $e(t)$ is the system error.

The nonlinear PD (NPD) controller has a similar structure as the linear PD controller (11), the NPD controller may be any control structure of the form

$$
u_{N}(t)=k_{p}(\cdot) e(t)+k_{d}(\cdot) \dot{e}(t)
$$

where $k_{p}(\cdot)$ and $k_{d}(\cdot)$ are the time-varying proportional and derivative gains, which may depend on system state, input or other variables.

Currently, several NPD controllers have been proposed for robotic application (Xu et.al., 1995; Kelly \& Ricardo, 1996; Seraji et.al., 1998). The NPD controller has superior trajectory tracking and disturbance rejection ability compared with the linear PD controllers for robot control. The NPD controller proposed by Han has a simple structure as (Han, 1994)

$$
u_{H}(t)=k_{p} f u n\left(e(t), \alpha_{1}, \delta_{1}\right)+k_{d} f u n\left(\dot{e}(t), \alpha_{2}, \delta_{2}\right)
$$

where the function fun can be defined as 


$$
f u n(x, \alpha, \delta)= \begin{cases}|x|^{\alpha} \operatorname{sign}(x), & |x|>\delta \\ x / \delta^{1-\alpha}, & |x| \leq \delta\end{cases}
$$

where $\alpha$ refers to the nonlinearity, specially the NPD will degenerate into the linear PD when $\alpha=1 ; \delta$ refers to the threshold of the error (or error derivative), and it is at the same magnitude with the error (or error derivative). The NPD controller (13) can be rewritten as the form (12), then $k_{p}(\cdot)$ can be derived as

$$
k_{p}(e)= \begin{cases}k_{p}|e|^{\alpha_{1}-1} & |e|>\delta_{1} \\ k_{p} \delta_{1}^{\alpha_{1}-1} & |e| \leq \delta_{1}\end{cases}
$$

Similarly, $k_{d}(\cdot)$ can be expressed as

$$
k_{d}(\dot{e})= \begin{cases}k_{d}|\dot{e}|^{\alpha_{2}-1} & |\dot{e}|>\delta_{2} \\ k_{d} \delta_{2}{ }^{\alpha_{2}-1} & |\dot{e}| \leq \delta_{2}\end{cases}
$$

In (15) and (16), $\alpha_{1}$ and $\alpha_{2}$ can be determined in the interval [0.5, 1.0] and [1.0, 1.5], respectively. This choice makes the nonlinear gains with the following characteristics (Han, 1994): on one hand, large gain for small error and small gain for large error; on the other hand, large gain for large error rate and small gain for small error rate. Such variations of the gains result in a rapid transition of the systems with favorable damping. In addition, the NPD controller is robust against the changes of the system parameters and the nonlinear factors. Thus the NPD controller (13) is suitable to the trajectory tracking of the high-speed planar parallel manipulator.

\subsection{Augmented NPD controller}

The augmented NPD (ANPD) controller developed here is designed by replacing the linear PD in the APD controller with the NPD algorithm. According to the APD controller and the NPD control algorithm (13), based on the dynamic model (10), the control law of the ANPD controller can be written as (Shang et.al., 2009)

$$
\tau_{A}=\mathbf{M}_{e} \ddot{\mathbf{q}}_{e}^{d}+\mathbf{C}_{e} \dot{\mathbf{q}}_{e}^{d}+\mathbf{S}^{T} \mathbf{f}_{a}+\mathbf{K}_{p}(\mathbf{e}) \mathbf{e}+\mathbf{K}_{d}(\dot{\mathbf{e}}) \dot{\mathbf{e}}
$$

where $\dot{\mathbf{q}}_{e}^{d}$ and $\ddot{\mathbf{q}}_{e}^{d}$ are the desired velocity and acceleration of the end-effector. The control law (17) can be divided into three terms according to different functions. The first term is the dynamics compensation defined by the desired trajectory, which can be written as

$$
\tau_{A 1}=\mathbf{M}_{e} \ddot{\mathbf{q}}_{e}^{d}+\mathbf{C}_{e} \dot{\mathbf{q}}_{e}^{d}
$$

The second term is the friction compensation, which can be written as

$$
\tau_{A 2}=\mathbf{S}^{T} \mathbf{f}_{a}
$$

The third term is the tracking error elimination, which can be written as

$$
\tau_{A 3}=\mathbf{K}_{p}(\mathbf{e}) \mathbf{e}+\mathbf{K}_{d}(\dot{\mathbf{e}}) \dot{\mathbf{e}}
$$


where $\mathbf{e}=\mathbf{q}_{e}^{d}-\mathbf{q}_{e}$ is the position error of the end-effector; $\mathbf{K}_{p}(\mathbf{e})$ and $\mathbf{K}_{d}(\dot{\mathbf{e}})$ are symmetric, positive definite matrices of time-varying gains. From (15) and (16), $\mathbf{K}_{p}(e)$ and $\mathbf{K}_{d}(\dot{e})$ can be expressed as

$$
\begin{aligned}
& \mathbf{K}_{p}(e)=\operatorname{diag}\left(k_{p}\left|x_{1}\right|^{\alpha_{1}-1}, k_{p}\left|x_{2}\right|^{\alpha_{1}-1}\right) \\
& \mathbf{K}_{d}(\dot{e})=\operatorname{diag}\left(k_{d}\left|y_{1}\right|^{\alpha_{2}-1}, k_{d}\left|y_{2}\right|^{\alpha_{2}-1}\right)
\end{aligned}
$$

where $k_{p}$ and $k_{d}$ are the positive constant gains. The variables $x_{i}, y_{i}, i=1,2$ are determined by the following rules: if $\left|e_{i}\right|>\delta_{1}$, then $x_{i}=e_{i}$, else $x_{i}=\delta_{1} ;$ if $\left|\dot{e}_{i}\right|>\delta_{2}$, then $y_{i}=\dot{e}_{i}$, else $y_{i}=\delta_{2} ; \alpha_{1}, \alpha_{2}, \delta_{1}$, and $\delta_{2}$ are the designed parameters which should be tuned in practice.

In the following, we will prove the asymptotic stability of the parallel manipulator system controlled by the ANPD controller (17). Firstly, we will introduce two lemmas (Kelly and Ricardo, 1996).

Lemma 1: Let $\alpha(\cdot)$ be a class $\mathrm{K}$ function and $f: \Re \rightarrow \Re$ a continuous function. If $f(x) \geq \alpha(|x|) \quad \forall x \in \mathfrak{R}$, then $\int_{0}^{x} f(\sigma) d \sigma>0, \forall x \neq 0 \in \mathfrak{R}$ and $\int_{0}^{x} f(\sigma) d \sigma \rightarrow \infty$ as $|x| \rightarrow \infty$.

Lemma 2: Consider the continuous diagonal matrix $K_{p}: \mathfrak{R}^{2} \rightarrow \mathfrak{R}^{2 \times 2}$

$$
K_{p}(e)=\left[\begin{array}{cc}
k_{p 1}\left(e_{1}\right) & 0 \\
0 & k_{p 2}\left(e_{2}\right)
\end{array}\right]
$$

Assume that there exist class $\mathrm{K}$ functions $\alpha_{i}(\cdot)$ such that

$$
x k_{p i}(x) \geq \alpha_{i}(|x|), x \in \Re, i=1,2
$$

then $\int_{0}^{\mathbf{e}} \boldsymbol{\xi}^{T} \mathbf{K}_{p}(\boldsymbol{\xi}) d \boldsymbol{\xi}>0, \forall \mathbf{e} \neq 0 \in \mathfrak{R}^{2}$, and $\int_{0}^{e} \xi^{T} \mathbf{K}_{p}(\xi) d \xi \rightarrow \infty$ as $|e| \rightarrow \infty$.

Next, we will give brief proof for Lemma 2 (Kelly and Ricardo, 1996). Define $f\left(e_{i}\right)=k_{p i}\left(e_{i}\right) e_{i}$, From Lemma 1, one can get

$$
\int_{0}^{e_{i}} f\left(\xi_{i}\right) d \xi_{i}>0, \quad \forall e_{i} \neq 0 \in \mathfrak{R}
$$

which is equivalent to

$$
\int_{0}^{e_{i}} k_{p i}\left(\xi_{i}\right) \xi_{i} d \xi_{i}>0, \quad \forall e_{i} \neq 0 \in \Re
$$

Therefore, the function $\int_{0}^{e} \xi^{T} \mathbf{K}_{p}(\xi) d \xi$ is positive definite. Also, Lemma 1 ensures that above integral is radically unbounded with respect to $\mathbf{e}$, and this implies $\int_{0}^{e} \xi^{T} \mathbf{K}_{p}(\xi) d \xi \rightarrow \infty$ as $|\mathbf{e}| \rightarrow \infty$.

Theorem 1: If the nonlinear gains $\mathbf{K}_{p}(\cdot)$ and $\mathbf{K}_{d}(\cdot)$ are defined by (19) and (20) respectively, the parallel manipulator system controlled by the ANPD control law (17) is asymptotically stable. 
Proof: Choose the Lyapunov function candidate as

$$
V(\mathbf{e}, \dot{\mathbf{e}})=\frac{1}{2} \dot{\mathbf{e}}^{T} \mathbf{M}_{e} \dot{\mathbf{e}}+\int_{0}^{\mathbf{e}} \boldsymbol{\xi}^{T} \mathbf{K}_{p}(\boldsymbol{\xi}) d \boldsymbol{\xi}
$$

where

$$
\int_{0}^{\mathbf{e}} \boldsymbol{\xi}^{T} \mathbf{K}_{p}(\boldsymbol{\xi}) d \boldsymbol{\xi}=\int_{0}^{e_{1}} \xi_{1} k_{p 1}\left(\xi_{1}\right) d \xi_{1}+\int_{0}^{e_{2}} \xi_{2} k_{p 2}\left(\xi_{2}\right) d \xi_{2}
$$

Considering the structural properties (a), the inertial matrix $\mathbf{M}_{e}$ is symmetric and positive definite matrix, thus the first term in (23) is positive definite. In addition, the integral term can be interpreted as a potential energy induced by the position error-driven part of the controller. Next, we will proof that the second term in (23) is positive definite. Considering $k_{p i}\left(e_{i}\right)$ is defined as

$$
k_{p i}\left(e_{i}\right)=\left\{\begin{array}{l}
k_{p i}\left|e_{i}\right|^{\alpha_{i}-1}, \quad\left|e_{i}\right|>\delta \\
k_{p i} \delta_{i}^{\alpha_{i}-1},\left|e_{i}\right| \leq \delta_{i}
\end{array}\right.
$$

Define class $\mathrm{K}$ functions $\alpha_{i}(\cdot)$ as

$$
\alpha_{i}\left(\left|e_{i}\right|\right)=\left\{\begin{array}{l}
\varepsilon_{i} e_{i}\left|e_{i}\right|^{\alpha_{i}-1},\left|e_{i}\right|>\delta_{1} \\
\varepsilon_{i} e_{i} \delta_{i}^{\alpha_{i}-1},\left|e_{i}\right| \leq \delta_{1}
\end{array} \quad \text {, and } k_{p i}>\varepsilon_{i}>0\right.
$$

With the Lemma 2, one can get the integral term in (23) is a radically unbounded positive definite function. Thus $V(\mathbf{e}, \dot{\mathbf{e}})$ is a positive function. Differentiating $V(t)$ with respect to time yields

$$
\dot{V}(\mathbf{e}, \dot{\mathbf{e}})=\dot{\mathbf{e}}^{T} \mathbf{M}_{e} \ddot{\mathbf{e}}+\frac{1}{2} \dot{\mathbf{e}}^{T} \dot{\mathbf{M}}_{e} \dot{\mathbf{e}}+\mathbf{e}^{T} \mathbf{K}_{p}(\mathbf{e}) \dot{\mathbf{e}}
$$

Combine the control law (17) and the dynamic model (10), the closed-loop system equation can be written as

$$
\mathbf{M}_{e} \ddot{\mathbf{e}}+\mathbf{C}_{e} \dot{\mathbf{e}}+\mathbf{K}_{p}(\cdot) \mathbf{e}+\mathbf{K}_{d}(\cdot) \dot{\mathbf{e}}=0
$$

Multiplying both sides of the above equation by $\dot{\mathbf{e}}^{T}$, and then substituting the resulting equation into (26) yields

$$
\dot{V}=-\dot{\mathbf{e}}^{T} \mathbf{K}_{d}(\cdot) \dot{\mathbf{e}}+\frac{1}{2} \dot{\mathbf{e}}^{T}\left(\dot{\mathbf{M}}_{e}-2 \mathbf{C}_{e}\right) \dot{\mathbf{e}}
$$

Considering the structural properties (b), then one can have $\dot{\mathbf{e}}^{T}\left(\dot{\mathbf{M}}_{e}-2 \mathbf{C}_{e}\right) \dot{\mathbf{e}}=0$ and

$$
\dot{V}=-\dot{\mathbf{e}}^{T} \mathbf{K}_{d}(\cdot) \dot{\mathbf{e}}
$$

As $\mathbf{K}_{d}(\cdot)$ is a symmetric, positive definite matrix, then $\dot{V}$ is a semi-negative definite matrix, thus the parallel manipulator system is stable. 
Now since $V(t) \geq 0$ and $\dot{V}(t) \leq 0, V(t)$ is bounded and decreasing, thus $V(t)$ converges to a limit. From the definition of $V(t)$, it implies that both $\mathbf{e}$ and $\dot{\mathbf{e}}$ are bounded. Since $\mathbf{M}_{e}$ is uniform positive definite, then $\mathbf{M}_{e}^{-1}$ exists and bounded, thus the closed-loop system equation (27) can be written as

$$
\ddot{\mathbf{e}}=-\mathbf{M}_{e}^{-1}\left(\mathbf{C}_{e} \dot{\mathbf{e}}+\mathbf{K}_{p}(\cdot) \mathbf{e}+\mathbf{K}_{d}(\cdot) \dot{\mathbf{e}}\right)
$$

So $\ddot{\mathbf{e}}$ is also bounded and $\ddot{V}(t)$ is bounded. Thus, $\dot{V}(t)$ is uniformly continuous. With the Barbalat Lemma (Slotine \& Li, 1991), one knows $\dot{\mathbf{e}} \rightarrow 0$ as $t \rightarrow \infty$, and this implies $\mathbf{e} \rightarrow 0$ as $t \rightarrow \infty$.

One can note that $\mathbf{\tau}_{A}$ in the control law (17) is the actuator torque of the task space, but in fact, we need the actuator torque $\boldsymbol{\tau}_{a}$ of the active joints. In practice, a solution that has a minimum weighted Euclidian norm is selected as the actual control input. The actual control input vector of the active joints can be written as

$$
\mathbf{\tau}_{a}=\left(\mathbf{S}^{T}\right)^{+}\left(\mathbf{M}_{e} \ddot{\mathbf{q}}_{e}^{d}+\mathbf{C}_{e} \dot{\mathbf{q}}_{e}^{d}+\mathbf{K}_{p}(\mathbf{e}) \mathbf{e}+\mathbf{K}_{d}(\dot{\mathbf{e}}) \dot{\mathbf{e}}\right)+\mathbf{f}_{a}
$$

where $\left(\mathbf{S}^{T}\right)^{+}=\mathbf{S}\left(\mathbf{S}^{T} \mathbf{S}\right)^{-1}$ is the pseudo-inverse of $\mathbf{S}^{T}$, satisfying $\mathbf{S}^{T}\left(\mathbf{S}^{T}\right)^{+}=\mathbf{I}$. For the parallel manipulator with redundant actuation, the singularity is eliminated in the effective workspace (Shang et.al., 2010). Thus, the pseudo-inverse matrix $\left(\mathbf{S}^{T}\right)^{+}$will not be close to the singularity for this parallel manipulator with redundant actuation.

\subsection{Nonlinear computed torque control}

An obvious drawback of the traditional CT controllers is the elimination of the tracking error by linear PD algorithm. However, the linear PD algorithm is not robust against the uncertain factors such as modeling error and nonlinear friction. To overcome this problem, the NPD algorithm can be combined with the conventional control strategies to improve the control accuracy. The NCT controller developed in this chapter is designed by replacing the linear PD in the CT controller with the NPD algorithm.

According to the NPD algorithm (13), based on the dynamic model (10), the control law of the NCT controller can be written as (Shang \& Cong, 2009)

$$
\mathbf{\tau}_{N}=\mathbf{M}_{e} \ddot{\mathbf{q}}_{e}^{d}+\mathbf{C}_{e} \dot{\mathbf{q}}_{e}+\mathbf{S}^{T} \mathbf{f}_{a}+\mathbf{M}_{e}\left(\mathbf{K}_{p}(\mathbf{e}) \mathbf{e}+\mathbf{K}_{d}(\dot{\mathbf{e}}) \dot{\mathbf{e}}\right)
$$

The control law (32) can be divided into three terms according to the different functions. The first term is the dynamics compensation defined by the desired acceleration and the actual velocity of the end-effector, which can be written as

$$
\mathbf{\tau}_{N 1}=\mathbf{M}_{e} \ddot{\mathbf{q}}_{e}^{d}+\mathbf{C}_{e} \dot{\mathbf{q}}_{e}
$$

The second term is the friction compensation, which can be written as

$$
\mathbf{\tau}_{N 2}=\mathbf{S}^{T} \mathbf{f}_{a}
$$

The third term is the tracking error elimination, which can be written as

$$
\mathbf{\tau}_{N 3}=\mathbf{M}_{e}\left(\mathbf{K}_{p}(\mathbf{e}) \mathbf{e}+\mathbf{K}_{d}(\dot{\mathbf{e}}) \dot{\mathbf{e}}\right)
$$


where $\mathbf{K}_{p}(\mathbf{e})$ and $\mathbf{K}_{d}(\dot{\mathbf{e}})$ are symmetric, positive definite matrices of time-varying gains. From (15) and (16), $\mathbf{K}_{p}(\mathbf{e})$ and $\mathbf{K}_{d}(\dot{\mathbf{e}})$ can be expressed as

$$
\begin{aligned}
& \mathbf{K}_{p}(\mathbf{e})=\operatorname{diag}\left(k_{p 1}\left|x_{1}\right|^{\alpha_{1}-1}, k_{p 2}\left|x_{2}\right|^{\alpha_{1}-1}\right) \\
& \mathbf{K}_{d}(\dot{\mathbf{e}})=\operatorname{diag}\left(k_{d 1}\left|y_{1}\right|^{\alpha_{2}-1}, k_{d 2}\left|y_{2}\right|^{\alpha_{2}-1}\right)
\end{aligned}
$$

where $k_{p i}, k_{d i}, i=1,2$ are positive constant gains. The variables $x_{i}, y_{i}$ are determined by the following rules: if $\left|e_{i}\right|>\delta_{1}$, then $x_{i}=e_{i}$, else $x_{i}=\delta_{1}$; if $\left|\dot{e}_{i}\right|>\delta_{2}$, then $y_{i}=\dot{e}_{i}$, else $y_{i}=\delta_{2}$. $\alpha_{1}, \alpha_{2}, \delta_{1}$, and $\delta_{2}$ are the designed parameters which should be tuned in practice.

In the following, the asymptotic stability of the parallel manipulator system controlled by the NCT controller (32) will be proven.

Theorem 2: If the nonlinear gains $\mathbf{K}_{p}(\cdot)$ and $\mathbf{K}_{d}(\cdot)$ are defined by (34) and (35) respectively, the parallel manipulator system controlled by the NCT controller (32) is asymptotically stable.

Proof: Choose the Lyapunov function candidate as

$$
V(\mathbf{e}, \dot{\mathbf{e}})=\frac{1}{2} \dot{\mathbf{e}}^{T} \dot{\mathbf{e}}+\int_{0}^{\mathbf{e}}|\xi|^{T} K_{p}(\xi) d \xi
$$

where $\int_{0}^{\mathbf{e}}|\boldsymbol{\xi}|^{T} \mathbf{K}_{p}(\boldsymbol{\xi}) d \boldsymbol{\xi}=\int_{0}^{e_{1}}\left|\xi_{1}\right| k_{p 1}\left(\xi_{1}\right) d \xi_{1}+\int_{0}^{e_{2}}\left|\xi_{2}\right| k_{p 2}\left(\xi_{2}\right) d \xi_{2}$. Obviously, the first term in (36) is positive definite. In addition, the integral term can be interpreted as the potential energy induced by the position error-driven part of the controller. Next, one can prove that the second term in (36) is positive definite. Considering $k_{p i}\left(e_{i}\right)$ is defined as

$$
k_{p i}\left(e_{i}\right)=\left\{\begin{array}{l}
k_{p i}\left|e_{i}\right|^{\alpha_{i}-1},\left|e_{i}\right|>\delta_{1} \\
k_{p i} \delta_{i}^{\alpha_{i}-1},\left|e_{i}\right| \leq \delta_{1}
\end{array}\right.
$$

and define class $\mathrm{K}$ functions $\alpha_{i}(\cdot)$ as

$$
\alpha_{i}\left(\left|e_{i}\right|\right)=\left\{\begin{array}{c}
\varepsilon_{i}\left|e_{i}\right|^{\alpha_{i}}, \quad\left|e_{i}\right|>\delta_{1} \\
\varepsilon_{i}\left|e_{i}\right| \delta_{i}^{\alpha_{i}-1},\left|e_{i}\right| \leq \delta_{1}
\end{array} \quad \text {, and } k_{p i}>\varepsilon_{i}>0\right.
$$

From (37) and (38), one knows $\left|e_{i}\right| k_{p i}\left(e_{i}\right) \geq \alpha_{i}\left(\left|e_{i}\right|\right)$. With the Lemma 2, one can get $\int_{0}^{e_{i}}\left|\xi_{i}\right|^{T} k_{p_{i}}\left(\xi_{i}\right) d \xi_{i}>0$, and $\int_{0}^{\mathbf{e}}|\xi|^{T} \mathbf{K}_{p}(\xi) d \xi \rightarrow \infty$ as $|\mathbf{e}| \rightarrow \infty$. So one can get the integral term in (36) is a radically unbounded positive definite function. Thus $V(\mathbf{e}, \dot{\mathbf{e}})$ is a positive definite function. Differentiating $V(\mathbf{e}, \dot{\mathbf{e}})$ with respect to time yields

$$
\dot{V}(\mathbf{e}, \dot{\mathbf{e}})=\dot{\mathbf{e}}^{T} \ddot{\mathbf{e}}+\mathbf{e}^{T} \mathbf{K}_{p}(\mathbf{e}) \dot{\mathbf{e}}
$$

Combine the control law (32) and the dynamic model (10) and consider $\mathbf{S}^{T} \boldsymbol{\tau}_{a}=\boldsymbol{\tau}_{N}$, the closed-loop system equation can be written as

$$
\mathbf{M}_{e}\left(\ddot{\mathbf{e}}+\mathbf{K}_{p}(\cdot) \mathbf{e}+\mathbf{K}_{d}(\cdot) \dot{\mathbf{e}}\right)=0
$$


Since $\mathbf{M}_{e}$ is uniform positive definite, then $\mathbf{M}_{e}^{-1}$ exists and bounded, thus the closed-loop system equation (40) can be written as

$$
\ddot{\mathbf{e}}+\mathbf{K}_{p}(\cdot) \mathbf{e}+\mathbf{K}_{d}(\cdot) \dot{\mathbf{e}}=0
$$

Multiplying both sides of the above equation by $\dot{\mathbf{e}}^{T}$, and then substituting the resulting equation into (39) yields

$$
\dot{V}=-\dot{\mathbf{e}}^{T} \mathbf{K}_{d}(\cdot) \dot{\mathbf{e}}
$$

As $\mathbf{K}_{d}(\cdot)$ is a symmetric, positive definite matrix, then $\dot{V}$ is a semi-negative definite matrix, thus the closed-loop system is stable. Considering the closed-loop equation (41) is autonomous system, and defining the region $\boldsymbol{\Omega}$ as

$$
\boldsymbol{\Omega}=\left\{\left[\begin{array}{c}
\mathbf{e} \\
\dot{\mathbf{e}}
\end{array}\right]: \dot{V}(\mathbf{e}, \dot{\mathbf{e}})=0\right\}=\left\{\left[\begin{array}{l}
\mathbf{e} \\
\dot{\mathbf{e}}
\end{array}\right]=\left[\begin{array}{c}
\mathbf{e} \\
0
\end{array}\right] \in \mathfrak{R}^{4}\right\}
$$

Thus $\left[\begin{array}{c}\mathbf{e} \\ \dot{\mathbf{e}}\end{array}\right]=\left[\begin{array}{c}0 \\ 0\end{array}\right]$ is the largest invariant set of $\boldsymbol{\Omega}=\left\{\left[\begin{array}{l}\mathbf{e} \\ \dot{\mathbf{e}}\end{array}\right]: \dot{V}(\mathbf{e}, \dot{\mathbf{e}})=0\right\}$, and constitutes an asymptotically stable equilibrium point. By using the LaSalle's theorem, one can get that the closed-loop system of the parallel manipulator is asymptotically stable.

\section{Nonlinear friction model and identification}

In this section, the friction compensation method based on a nonlinear friction model is developed for the parallel manipulator. This nonlinear friction model enables reconstruction of viscous, Coulomb, and Stribeck friction effects of the parallel manipulator. Identification experiments are carried out, and parameters in the nonlinear friction model are estimated by nonlinear optimization.

\subsection{Nonlinear friction modeling}

In order to reconstruct the nonlinear friction effect, the nonlinear friction model can be formulated as (Hensen et.al., 2000; Kostic et.al., 2004)

$$
f(\dot{\theta})=B_{v} \dot{\theta}+\sum_{k=1}^{3} f_{k}\left(1-\frac{2}{1+e^{2 \omega_{k}} \dot{\theta}}\right), \quad k=1,2,3
$$

where the first term represents the viscous friction and $B_{v}$ is the viscous friction coefficient. The other terms model the Coulomb and Stribeck friction effects. The parameters $f_{k}$ represent the magnitude of the Coulomb friction and the Stribeck curve. The parameters $\omega_{k}$ determine the slope in the approximation of the sigmoid function in the Coulomb friction and the Stribeck curve.

Obviously, the nonlinear friction model is an odd continuous function. Since $f(\dot{\theta})$ is clearly zero at $\theta=0$, the model does not capture the static friction. The friction model does not describe stiction, because the system will always slide for an applied force unequal to zero. The stiction regime will be approximated, if the slope of the function near $\dot{\theta}=0$ is very steep. Then the model can still give acceptable simulation results, i.e., angular displacement 
during stiction is neglectable. On the other hand, a continuous friction function will facilitate the numerical solution if such a model is used in parameter identification.

In (44), there are three sigmoid functions. If more sigmoid functions are selected, the estimation accuracy with this model will be better, but the friction model will have more parameters and it will be more complicated. So for this nonlinear friction model, a suitable number of the sigmoid function is important. One can also analyze this problem with the neural network. The nonlinear terms in (44) can be constructed with a two layers neural network, i.e., one hidden layer and one output layer. Defining the weight matrices for the first and second layer as $\mathbf{W}_{1}$ and $\mathbf{W}_{2}$, the neural network output can be written as (Hensen et.al., 2000)

$$
f^{\prime}(\dot{\theta})=\mathbf{W}_{2}^{T} \sum\left(\mathbf{W}_{1} \dot{\boldsymbol{\theta}}+b_{1}\right)+b_{2}
$$

where $b_{i}$ represents the bias value for the neurons in the $i$-th layer and $\sum(\cdot)$ is a nonlinear operator with $\sum(x)=\left[\begin{array}{lll}\sigma\left(x_{1}\right) & \sigma\left(x_{2}\right) & \sigma\left(x_{3}\right)\end{array}\right]^{T}$, the activation function $\sigma(x)=1-\frac{2}{1+e^{2 x}}$. From (44), the parameter b1 and b2 are both zero. The weight matrix for the first layer and the second layer can be written as $\mathbf{W}_{1}=\left[\begin{array}{lll}\omega_{1} & \omega_{2} & \omega_{3}\end{array}\right]^{T}$ and $\mathbf{W}_{2}=\left[\begin{array}{lll}f_{1} & f_{2} & f_{3}\end{array}\right]^{T}$ respectively. As we known, increasing the number of the hidden neurons, the approximation performance with the network will be better. However, too many hidden neurons will make the network more complicated and the training time may be longer. In practice, suitable number of the hidden neurons should be selected. In order to model the nonlinear friction of the parallel manipulator, two hidden neurons are enough, that is to say two sigmoid functions will be selected.

If the friction of the passive joints is neglected, according to (44), one can define the nonlinear friction model for the 2-DOF planar parallel manipulator as follows

$$
f_{a i}=B_{v i} \dot{q}_{a i}+f_{1 i}\left(1-\frac{2}{1+e^{2 \omega_{1 i} \dot{q}_{a i}}}\right)+f_{2 i}\left(1-\frac{2}{1+e^{2 \omega_{2 i} \dot{q}_{a i}}}\right)+d_{i}, i=1,2,3
$$

where the first term represents the viscous friction and $B_{v i}$ is the viscous friction coefficient of the $i$ th active joint; $d_{i}$ represents the zero drift of the motion control board; the remaining terms model the Coulomb and Stribeck friction effects of the $i$ th active joint. The parameter $f_{1 i}$ and $f_{2 i}$ represent the magnitude of the Coulomb friction and the Stribeck curve. The parameters $\omega_{1 i}$ and $\omega_{2 i}$ determine the slope in the approximation of the sigmoid function in the Coulomb friction and the Stribeck curve.

\subsection{Nonlinear friction identification}

In the dynamic model Eq. (10), the dynamic parameters can be calculated directly, and only the parameters in the nonlinear friction model Eq. (46) need to be identified. In Eq. (10), the mass, length and joint angles all united into the standard units. The corresponding torque has the unit N.m. Since the commanded torque for the motion control board of the parallel manipulator is digital value, the proportion should be obtained between the torque of the unit N.m and the commanded digital value of the torque (Shang et.al., 2008). Defining the dynamic torque $\left(\mathbf{S}^{T}\right)^{+}\left(\mathbf{M}_{e} \ddot{\mathbf{q}}_{e}+\mathbf{C}_{e} \dot{\mathbf{q}}_{e}\right)=\mathbf{D}$ and the proportion is $k$, the dynamic model Eq. (10) can be rewritten as 


$$
\mathbf{D} \cdot k+\mathbf{f}_{a}=\mathbf{\tau}_{a}
$$

Substituting the nonlinear friction model (46) into (47), one can define the optimization function $J$ as follows

$$
J=\sum_{j=1}^{N} \sum_{i=1}^{3}\left(\tau_{a i}^{j}-k\left(D_{i}^{j}\right)-\left(B_{v i} \dot{q}_{a i}^{j}+f_{1 i}\left(1-\frac{2}{1+e^{2 \omega_{1 i} \dot{q}_{a i}^{j}}}\right)+f_{2 i}\left(1-\frac{2}{1+e^{2 \omega_{2 i} \dot{q}_{a i}^{j}}}\right)+d_{i}\right)\right)^{2}
$$

where $\tau_{a i}^{j}$ and $D_{i}^{j}$ represent the actuator torque and the dynamic torque of the $i$ th active joint in the $j$ th configuration respectively. And $\dot{q}_{a i}^{j}$ represents the velocity of the $i$ th active joint in the $j$ th configuration.

The parameters $B_{v i}, f_{1 i}, f_{2 i}, \omega_{1 i}, \omega_{2 i}, d_{i}$, and the proportion $k$, a total of 19 parameters, are selected as the optimization variables. These parameters will be estimated by making the optimization function $J$ minimum. Parameter optimization procedures are programmed with Matlab, and the nonlinear optimization function fmincon finding a constrained minimum of a function of several variables is called for in Matlab. In order to use the fmincon, the first step is set the initial value, the lower limit value and the upper limit value of the 19 optimization variables. Then (48) is defined as the optimized function of fmincon. The third step is getting the variables $\tau_{a i .}^{j}, D_{i}^{j}$, and $\dot{q}_{a i}^{j}$ in (48). Next we will give the procedures about getting the variables $\tau_{a i}^{j}, D_{i}^{j}$, and $\dot{q}_{a i}^{j}$ in our actual identification experiment.

In actual identification experiment, the end-effector of the parallel manipulator is driven to track a circular trajectory. The center coordinates of the circle are $(0.29,0.25)$ and radius is 0.07 , the unit is meter, this circle motion is repeated clockwise for 15 times. The parallel manipulator is controlled by the PD controller in the task space, the actuator torque is also the control input, thus $\tau_{a i}^{j}$ is a variable known. The control input is selected in the nullspace of the matrix $\mathbf{S}^{T}$, thus the actuator torque $\tau_{a i}^{j}$ in (48) is also in the null-space of the matrix $\mathbf{S}^{T}$. And this selection will make the control input used in the identification experiment minimum. For the parallel manipulator, only angles of the active joints can be measured directly by the absolute optical-electrical encoders. The angular velocity of the active joints is obtained by numerical differentiation of the active joint angles, and a lowpass filter is adopted to filter the angular velocity signal, then we will get the variable $\dot{q}_{a i}^{j}$. The angular acceleration of the joints is obtained by numerical differentiation of the filtered angular velocity. With the velocity and the acceleration of the joints, and considering the kinematics of the parallel manipulator, the actual velocity and acceleration of the endeffector can be obtained. Thus $D_{i}^{j}$ can be calculated with these variables. With the actual values of the variable $\tau_{a i}^{j}, D_{i}^{j}$, and $\dot{q}_{a i}^{j}$, the unknown parameters of the parallel manipulator are identified and results are shown in Table 1.

\subsection{Coulomb + viscous friction identification}

In order to compare with the nonlinear friction model, a common Coulomb + viscous friction model containing the viscous friction and Coulomb friction effect is established for the parallel manipulator. The friction model can be written as

$$
f_{a i}=\operatorname{sign}\left(\dot{q}_{a i}\right) f_{c i}+f_{v i} \dot{q}_{a i}+d_{i}, i=1,2.3
$$

where $f_{c i}$ represents the Coulomb friction; $f_{v i}$ represents the coefficient of the viscous friction; $d_{i}$ represents the zero drift of the motion control board. 


\begin{tabular}{|c|c|c|c|c|c|c|c|}
\hline parameters & values & parameters & values & parameters & values & parameters & values \\
\hline$k$ & 508.7 & $\omega_{21}$ & -16.4 & $\omega_{12}$ & -18 & $f_{23}$ & 500 \\
\hline$B_{v 1}$ & 892.8 & $d_{1}$ & -2.6 & $\omega_{22}$ & 5.1 & $\omega_{13}$ & -0.9 \\
\hline$f_{11}$ & 1040.1 & $B_{v 2}$ & 1396.4 & $d_{2}$ & -21.9 & $\omega_{23}$ & 10 \\
\hline$f_{21}$ & -268.0 & $f_{12}$ & -309.0 & $B_{v 3}$ & 402.6 & $d_{3}$ & 30 \\
\hline$\omega_{11}$ & 0.7 & $f_{22}$ & -61.8 & $f_{13}$ & -867.7 & & \\
\hline
\end{tabular}

Table 1. Identification results of the nonlinear friction model.

With the analysis of the identification of the nonlinear friction model, the corresponding work of the Coulomb + viscous friction model is much simpler. Substituting the Coulomb + viscous friction model (49) into (47), one can get a linear equation about the identified parameters as follows

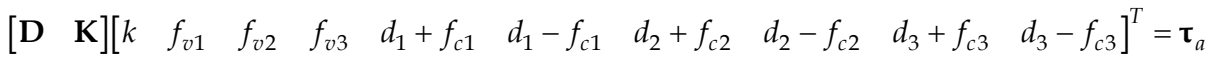

where

$$
\mathbf{K}=\left[\begin{array}{ccccccccc}
\dot{q}_{a 1} & 0 & 0 & u_{1} & l_{1} & 0 & 0 & 0 & 0 \\
0 & \dot{q}_{a 2} & 0 & 0 & 0 & u_{2} & l_{2} & 0 & 0 \\
0 & 0 & \dot{q}_{a 3} & 0 & 0 & 0 & 0 & u_{3} & l_{3}
\end{array}\right]
$$

For simplicity, parameter combinations $d_{i}+f_{c i}$ and $d_{i}-f_{c i}$ are viewed as identified parameters, and the coefficients $u_{i}$ and $l_{i}$ of the parameters are determined by the following rules: $u_{i}=1, l_{i}=0$ when $\dot{q}_{a i} \geq 0$, and $u_{i}=0, l_{i}=1$ when $\dot{q}_{a i}<0$.

There are 10 parameters to be identified in Eq. (50), but only three independent equations can be got for each sampling point. So a group of linear equations about the unknown parameters can be got with the sampling data of a continuous trajectory, then the Least Squares method is used to identify the unknown parameters.

The identification experiment designed for the Coulomb + viscous friction model is the same with the nonlinear friction model discussed in section 4.2. Identification results of the Coulomb + viscous friction model are shown in Table 2 .

\begin{tabular}{|c|c|c|c|}
\hline parameters & values & parameters & values \\
\hline$k$ & 512.7 & $d_{1}-f_{c 1}$ & -261.7 \\
\hline$f_{v 1}$ & 1534.8 & $d_{2}+f_{c 2}$ & 212.6 \\
\hline$f_{v 2}$ & 1415.9 & $d_{2}-f_{c 2}$ & -256 \\
\hline$f_{v 3}$ & 1475.1 & $d_{3}+f_{c 3}$ & 179.2 \\
\hline$d_{1}+f_{c 1}$ & 248.5 & $d_{3}-f_{c 3}$ & -129 \\
\hline
\end{tabular}

Table 2. Identification results of the Coulomb + viscous friction model

\section{Experiments}

As shown in Fig. 2, the actual experiment platform is a 2-DOF parallel manipulator with redundant actuation designed by Googol Tech. Ltd. in Shenzhen, China. It is equipped with 
three permanent magnet synchronous servo motors with harmonic gear drives. The active joint angles are measured with absolute optical-electrical encoders. The nonlinear dynamic controllers and the friction compensation method are programmed with the Visual $\mathrm{C}++$, and the algorithms run on a Pentium III CPU at 733MHz. with the sampling period $2 \mathrm{~ms}$.

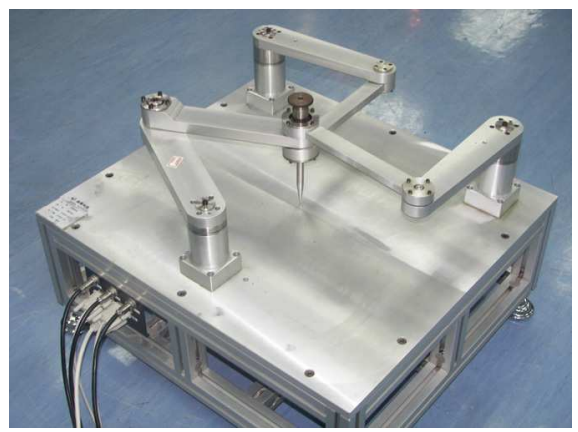

Fig. 2. The prototype of the 2-DOF parallel manipulator with redundant actuation

\subsection{Experiments of the ANPD controller}

The trajectory tracking control experiment is designed for the parallel manipulator to validate the ANPD controller. The desired trajectory of the end-effector is a straight line, the starting point is $(0.22,0.29)$ and the ending point is $(0.37,0.21)$, thus the motion distance is $0.17 \mathrm{~m}$. The profile of the desired velocity is an S-type curve (Cheng et.al., 2003). In the experiment, the low-speed and high-speed motions are both tested. For the low-speed motion, the max velocity is $0.2 \mathrm{~m} / \mathrm{s}$, the max acceleration is $5 \mathrm{~m} / \mathrm{s}^{2}$, and the jerk is $200 \mathrm{~m} / \mathrm{s}^{3}$. For the high-speed motion, the max velocity is $0.5 \mathrm{~m} / \mathrm{s}$, the max acceleration is $10 \mathrm{~m} / \mathrm{s}^{2}$, and the jerk is $400 \mathrm{~m} / \mathrm{s}^{3}$.

In order to implement the ANPD controller (17), the dynamic parameters in (18.a) and the friction parameters in (18.b) must be known. In the experiment, the nominal values of the dynamic parameters are used (Shang et.al., 2008). Then, with the known dynamic parameters, the friction parameters in the Coulomb + viscous friction model can be identified by the Least Squares method, as shown in Table 2. In fact, the control parameters in (18.c) are tuned and determined by the actual experiments. The procedures to tune the control parameters in (18.c) can be summarized as follows:

1. Assume $k_{p 1}=k_{p 2}=k_{p}, k_{d 1}=k_{d 2}=k_{d}$. Let $k_{d}=0, \alpha_{1}=1, \alpha_{2}=1$, and increase the value of $k_{p}$ from zero until the system show a little oscillation to some extent.

2. Keep the value of $k_{p}$ tuned well in the first stage, and increase the value of $k_{d}$ to improve the dynamic performance further.

3. Regulate finely the above two values and make tradeoffs between $k_{p}$ and $k_{d}$.

4. Find the maximum error and error rate of the end-effector under the tuned value of $k_{p}$ and $k_{d}$.

5. In the ANPD controller, $\delta_{1}$ and $\delta_{2}$ are the threshold of the error and the error rate. If $\delta_{1}$ is tuned bigger than the maximum error, then the proportional gain $k_{p}\left(e_{i}\right)$ will always equals to $k_{p} \delta_{1}^{\alpha_{1}-1}$; and $\delta_{1}$ is tuned close to 0 , then $k_{p}\left(e_{i}\right)$ will always equal to $k_{p}\left|e_{i}\right|^{\alpha_{1}-1}$. So, $\delta_{1}$ should be made a tradeoff between the maximum error and 0 error. Similar method can be used to tune parameter $\delta_{2}$. From our actual experiences, the 
value of $\delta_{1}$ is tuned to the half value of the maximum error, and the value of $\delta_{2}$ is tuned to the half of the maximum error rate. This choice has good control performance and it's easy to implement.

6. For the parameters $\alpha_{1}=1$ and $\alpha_{2}=1$, the proportional gain $k_{p}\left(e_{i}\right)$ is a constant of $k_{p}$, and the derivative gains $k_{d}\left(\dot{e}_{i}\right)$ is a constant of $k_{d}$. Thus the NPD algorithm can be considered as the linear PD algorithm. So decrease the value of $\alpha_{1}\left(0.5 \leq \alpha_{1} \leq 1\right)$, and decrease the value of $k_{p}$ at the same time to improve the error curve further, and make tradeoffs between the two values. Using this step, one can get the nonlinear proportional gain of the ANPD controller.

7. Increase the value of $\alpha_{2}\left(1 \leq \alpha_{2} \leq 1.5\right)$, and decrease the value of $k_{d}$ at the same time to improve the error rate curve further, then make tradeoffs between the two values.

Using the above procedures, the ANPD controller parameters are tuned as follow:

$$
k_{p}=4500, k_{d}=470, \delta_{1}=3 \times 10^{-4}, \delta_{2}=3 \times 10^{-3}, \alpha_{1}=0.7, \alpha_{2}=1.1
$$

In order to make a comparison between the ANPD controller and the APD controller, the same tracking experiments are implemented on the parallel manipulator. We choose the APD controller is because it has nonlinear dynamics compensation and friction compensation. In the APD controller, the control input vector of the three actuated joints can be calculated as (Shang et.al., 2009)

$$
\mathbf{\tau}_{a}=\left(\mathbf{S}^{T}\right)^{+}\left(\mathbf{M}_{e} \ddot{\mathbf{q}}_{e}^{d}+\mathbf{C}_{e} \dot{\mathbf{q}}_{e}^{d}+\mathbf{K}_{l p} \mathbf{e}+\mathbf{K}_{l d} \dot{\mathbf{e}}\right)+\mathbf{f}_{a}
$$

where $\mathbf{K}_{l p}$ and $\mathbf{K}_{l d}$ are both symmetric, positive definite matrices of constant gains. In the APD controller (51), $\mathbf{M}_{e}$ and $\mathbf{C}_{e}$ can be calculated with the nominal dynamic parameters, and $\mathbf{f}_{a}$ can be calculated with the values of the friction parameters shown in Table 2 . The procedures of tuning parameters $\mathbf{K}_{l p}$ and $\mathbf{K}_{l d}$ in APD controller are similar to the procedures of tuning parameters $k_{p}$ and $k_{d}$ in ANPD controller. Thus, the tuning procedures (1) to (3) can be used to tune the parameters $\mathbf{K}_{l p}$ and $\mathbf{K}_{l d}$.

The experiment results of the APD and ANPD controller are shown in Fig. 3-4. Fig. 3a and Fig. $3 \mathrm{~b}$ are the tracking errors of the end-effector at the low-speed on the X-direction and $\mathrm{Y}$ direction respectively. From the experimental curves, one can see that the ANPD controller can decrease the tracking errors during the whole motion process obviously, and the maximum error in the motion is smaller. Fig. $4 \mathrm{a}$ and Fig. $4 \mathrm{~b}$ are the tracking errors of the end-effector at the high-speed on the X-direction and Y-direction respectively. One can find that the tracking errors are much smaller with the ANPD controller than with the APD controller, especially at the acceleration process. And one can conclude that, by the ANPD controller, the performance improvement of trajectory tracking accuracy at the high-speed is more obvious than at the low-speed.

Furthermore, to evaluate the performances of the two controllers, the root-square mean error (RSME) of the end-effector position is selected as the performance index

$$
\begin{aligned}
\text { RSME } & =\sqrt{\frac{1}{N} \sum_{j=1}^{N}\left(e_{x}^{2}(j)+e_{y}^{2}(j)\right)} \\
& =\sqrt{\frac{1}{N} \sum_{j=1}^{N}\left(\left(x^{d}(j)-x(j)\right)^{2}+\left(y^{d}(j)-y(j)\right)^{2}\right)}
\end{aligned}
$$




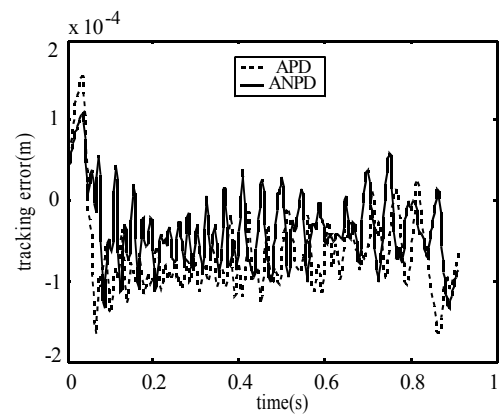

(a)

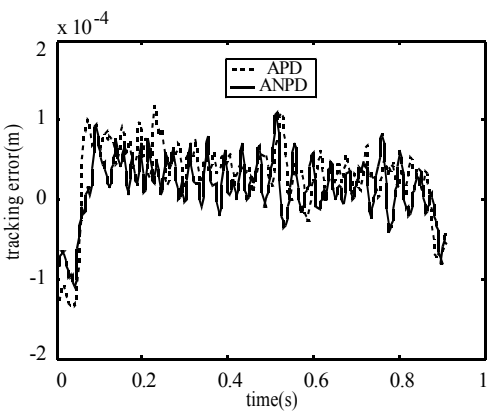

(b)

Fig. 3. Tracking errors of the end-effector at the low-speed: (a) X-direction; (b) Y-direction

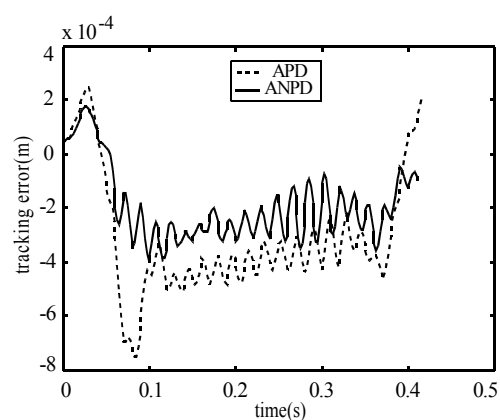

(a)

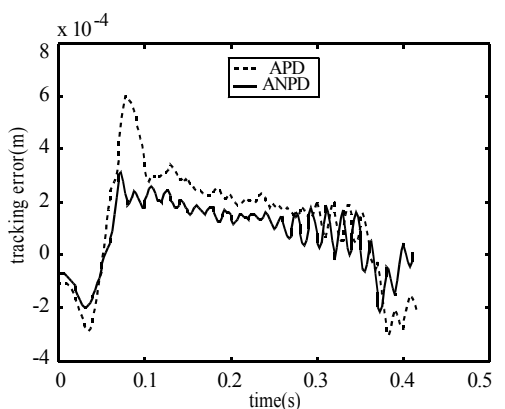

(b)

Fig. 4. Tracking errors of the end-effector at the high-speed: (a) X-direction; (b) Y-direction.

where $x^{d}(j)$ and $y^{d}(j)$ represent the $\mathrm{X}$-direction and Y-direction position coordinates at the $j$ th sampling point of the desired trajectory respectively; $x(j)$ and $y(j)$ represent the $X$ direction and Y-direction position coordinates of the $j$ th sampling point of the actual trajectory respectively.

The RSME results of the trajectory tracking experiment of the ANPD and APD controller are shown in Table 3. From the data of the RPE (reduced percentage of error) in Table 3, the ANPD controller can increase the position accuracy of the end-effector above $30 \%$, compared with the conventional APD controller.

\begin{tabular}{|c|c|c|}
\hline & at slow-speed $(\mathrm{m})$ & at high-speed $(\mathrm{m})$ \\
\hline APD & $1.04 \times 10^{-4}$ & $4.55 \times 10^{-4}$ \\
\hline ANPD & $7.22 \times 10^{-5}$ & $2.78 \times 10^{-4}$ \\
\hline RPE & $30.6 \%$ & $38.9 \%$ \\
\hline
\end{tabular}

Table 3. RSME of the APD and ANPD controller

\subsection{Experiments of the NCT controller}

In order to validate the NCT controller further, the trajectory tracking control experiment is designed for the parallel manipulator. Both the linear and circular trajectories in the 
workspace are selected as the desired trajectory. For the linear trajectory, the starting point is $(0.22,0.19)$ and the ending point is $(0.35,0.29)$, thus the motion distance is $0.164 \mathrm{~m}$. The velocity profile of the linear trajectory is an S-type curve (Cheng et.al., 2003), the max velocity is $0.5 \mathrm{~m} / \mathrm{s}$, the max acceleration is $10 \mathrm{~m} / \mathrm{s}^{2}$, and the jerk is $400 \mathrm{~m} / \mathrm{s}^{3}$. For the circular trajectory with the constant speed of $0.5 \mathrm{~m} / \mathrm{s}$, the center is $(0.29,0.25)$ and the starting point is $(0.29,0.31)$, thus the radius is $0.06 \mathrm{~m}$.

The actual implement of the NCT controller is similar to the ANPD controller, and the dynamic parameters in (33.a) and the friction parameters in (33.b) must be known. In the experiment, the nominal values are selected as the values of the actual dynamic parameters (Shang et.al., 2008). Then, with the known dynamic parameters, the friction parameters can be identified by the Least Squares method (Shang et.al., 2008). And the values of the control parameters in (33.c) are tuned and determined by the actual experiments. The tuning procedures for the ANPD controller can be used to tune the NCT controller. Using those procedures, the NCT controller parameters are tuned as follows: $k_{p}=2400, k_{d}=240$, $\delta_{1}=3 \times 10^{-4}, \delta_{2}=3 \times 10^{-3}, \alpha_{1}=0.7, \alpha_{2}=1.1$. Moreover, to demonstrate that the NCT controller can improve the tracking accuracy of the end-effector, experiments using the CT controller are carried out as comparison (Shang \& Cong, 2009). The CT controller is chosen because it has friction compensation and feedback dynamics compensation. In the CT controller, the control input vector of the three active joints can be calculated as

$$
\mathbf{\tau}_{a}=\left(\mathbf{S}^{T}\right)^{+}\left(\mathbf{M}_{e} \ddot{\mathbf{q}}_{e}^{d}+\mathbf{C}_{e} \dot{\mathbf{q}}_{e}+\mathbf{M}_{e}\left(\mathbf{K}_{l p} e+\mathbf{K}_{l d} \dot{e}\right)\right)+\mathbf{f}_{a}
$$

where $\mathbf{K}_{l p}$ and $\mathbf{K}_{l d}$ are both symmetric, positive definite matrices of constant gains.

In the CT controller (53), the dynamic parameters in $\mathbf{M}_{e}$ and $\mathbf{C}_{e}$, and the friction parameters in $\mathbf{f}_{a}$ are the same with these of the NCT controller. The procedures of tuning parameters of $\mathbf{K}_{l p}$ and $\mathbf{K}_{l d}$ in the CT controller are similar to the procedures of tuning parameters of $k_{p}$ and $k_{d}$ in the NCT controller. Thus, the tuning procedures (1), (2), and (3) can be used to tune the parameters of $\mathbf{K}_{l p}$ and $\mathbf{K}_{l d}$. Using the above methods, the CT controller parameters are tuned as follows: $\mathbf{K}_{l p}=\operatorname{diag}(20000,20000)$, $\mathbf{K}_{l d}=\operatorname{diag}(150,150)$.

The tracking error curves of the end-effector controlled by the CT and NCT controller are shown in Fig. 5-6. Fig. 5 is the linear trajectory tracking errors of the end-effector on the Xdirection and Y-direction. From the experiment curves, one can see that the NCT controller can decrease the tracking errors during the whole motion process obviously, and the maximum error in the motion is smaller. Fig. 6 is the circular trajectory tracking errors of the end-effector on the X-direction and Y-direction. From the curves one can see, the tracking accuracy is improved obviously using the NCT controller, compared with the CT controller. The RSME results of the trajectory tracking experiment of the NCT and CT controller are shown in Table 4. From the data of the RPE in Table 4, the NCT controller can increase the position accuracy of the end-effector above $35 \%$, compared with the conventional CT controller.

\begin{tabular}{|c|c|c|}
\hline & Line $(\mathrm{m})$ & Circle $(\mathrm{m})$ \\
\hline CT & $4.77 \times 10^{-4}$ & $4.41 \times 10^{-4}$ \\
\hline NCT & $3.08 \times 10^{-4}$ & $2.59 \times 10^{-4}$ \\
\hline RPE & $35.4 \%$ & $41.3 \%$ \\
\hline
\end{tabular}

Table 4. RSME of the CT and NCT controller 


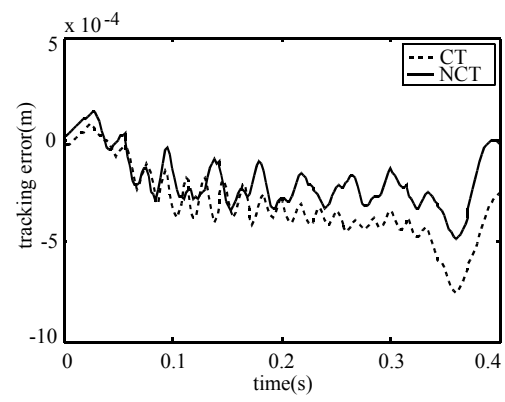

(a)

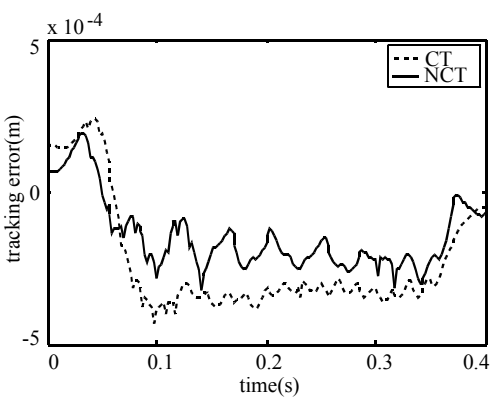

(b)

Fig. 5. Linear trajectory tracking errors of the end-effector: (a) X-direction; (b) Y-direction

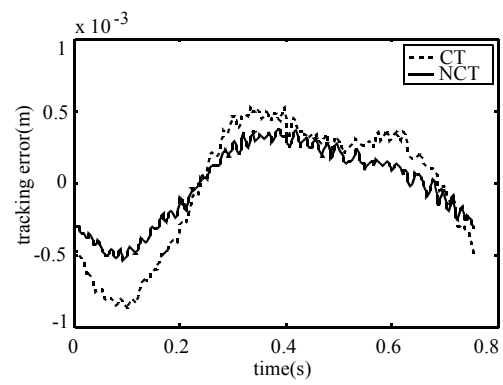

(a)

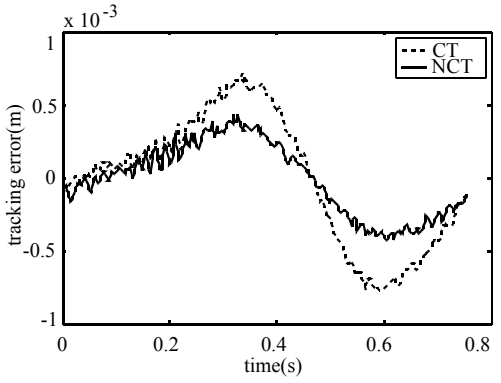

(b)

Fig. 6. Circular trajectory tracking errors of the end-effector: (a) X-direction; (b) Y-direction.

\subsection{Experiments of nonlinear friction compensation}

In order to compare with the compensation performances of the nonlinear friction model and the Coulomb + viscous friction model, the trajectory tracking experiments are implemented on the parallel manipulator. In the actual experiment, the augmented PD (APD) controller is designed in the task space for the parallel manipulator (Shang et.al., 2009). In the APD controller, the control input vector of the three active joints can be calculated as

$$
\mathbf{\tau}_{a}=\left(\mathbf{S}^{T}\right)^{+}\left(\mathbf{M}_{e} \ddot{\mathbf{q}}_{e}^{d}+\mathbf{C}_{e} \dot{\mathbf{q}}_{e}^{d}+\mathbf{K}_{l p} \mathbf{e}+\mathbf{K}_{l d} \dot{\mathbf{e}}\right)+\mathbf{f}_{a}
$$

where the term $\mathbf{f}_{a}$ is the friction compensation calculated by the nonlinear friction model (46) with the parameter values in Table 1 . Moreover, $\mathbf{f}_{a}$ can be calculated by the Coulomb + viscous friction model (49) with the parameter values in Table 2. If the term $\mathbf{f}_{a}$ is neglected in the APD controller (54), it means the friction compensation is not considered in the controller and the friction is ignored in the parallel manipulator.

Both the straight line and the circle in the task space are selected as the desired trajectory to study the friction compensation. For the straight line, the starting point is $(0.22,0.29)$ and the ending point is $(0.37,0.21)$, thus the motion distance is $0.17 \mathrm{~m}$. The profile of the desired velocity is trapezoidal curve. In the experiment, both the low-speed and high-speed motions are implemented. For the low-speed motion, the maximum velocity is $0.2 \mathrm{~m} / \mathrm{s}$ and the acceleration is $5 \mathrm{~m} / \mathrm{s}^{2}$. For the high-speed motion, the maximum velocity is $0.5 \mathrm{~m} / \mathrm{s}$ and the 
acceleration is $10 \mathrm{~m} / \mathrm{s}^{2}$. In the circle motion with constant speed, the center coordinates of the circle are $(0.29,0.25)$ and radius is 0.04 , also both the low-speed motion of $0.2 \mathrm{~m} / \mathrm{s}$, and the high-speed motion of $0.5 \mathrm{~m} / \mathrm{s}$ are implemented.

Linear trajectory tracking errors of the end-effector at the slow-speed and the high-speed are shown in Fig.7. From the curves one can see, the tracking errors are much smaller with the friction compensation methods based on the Coulomb + viscous model or the nonlinear model, compared with the without friction compensation method which means the term $\mathbf{f}_{a}$ is neglected in the APD controller (54). Especially, the maximum error at the acceleration process is decreased greatly with the friction compensation methods. Also one can see that, compared with the friction compensation based on the Coulomb + viscous model, the tracking accuracy has improved further at the low-speed, and the improvement at the high-speed is even more apparent by using the friction compensation based on the nonlinear model.

Circular trajectory tracking errors of the end-effector at the slow-speed and the high-speed are shown in Fig.8. From the curves one can see, the tracking accuracy is improved obviously using the two friction compensation methods, compared with the without friction compensation method. Also one can see that, with the friction compensation based on the nonlinear model, the tracking error is decreased at the low-speed, and the improvement at the high-speed is more obvious than the friction compensation based on the Coulomb + viscous.

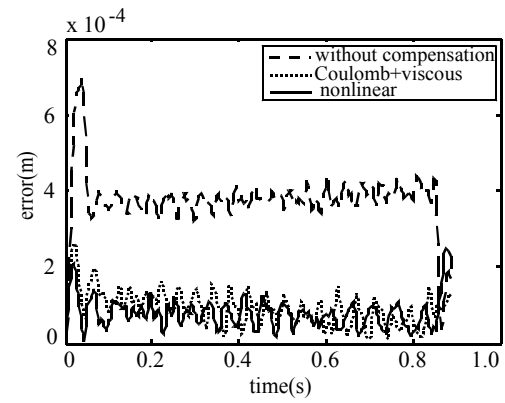

(a)

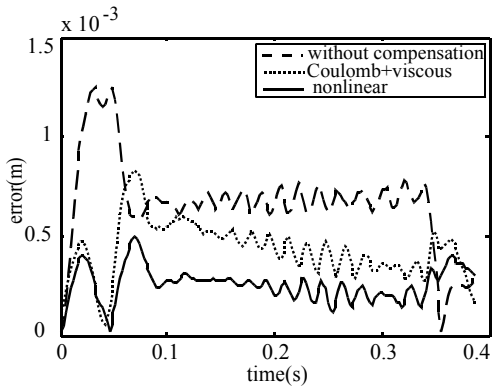

(b)

Fig. 7. Linear trajectory tracking error of the end-effector: (a) at the low-speed; (b) at the high-speed.

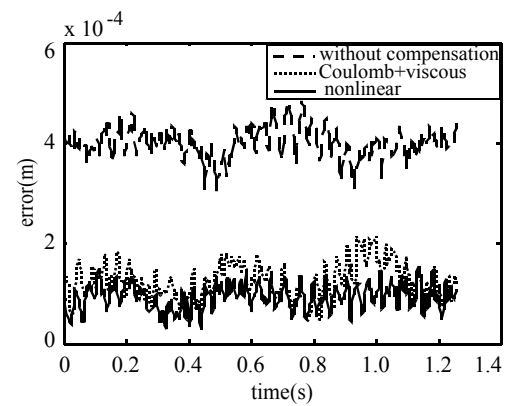

(a)

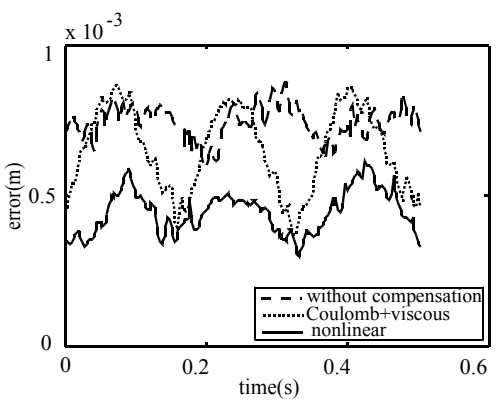

(b)

Fig. 8. Circular trajectory tracking error of the end-effector: (a) at the low-speed; (b) at the high-speed 
Furthermore, the RSMEs of the trajectory tracking experiment are shown in Table 5. From the data in the table one can see, by using the two friction compensation methods, the RSMEs are much smaller than the method ignoring friction compensation. And the RSMEs of the friction compensation based on the nonlinear model are smaller than the friction compensation with the Coulomb + viscous model, especially when the speed is higher.

\begin{tabular}{|c|c|c|c|c|}
\hline \multirow{2}{*}{$\begin{array}{c}\text { Friction compensation } \\
\text { method }\end{array}$} & \multicolumn{2}{|c|}{ Straight line motion $(\mathrm{m})$} & \multicolumn{2}{c|}{ Circle motion $(\mathrm{m})$} \\
\cline { 2 - 5 } & $0.2 \mathrm{~m} / \mathrm{s}$ & $0.5 \mathrm{~m} / \mathrm{s}$ & $0.2 \mathrm{~m} / \mathrm{s}$ & $0.5 \mathrm{~m} / \mathrm{s}$ \\
\hline Without compensation & $3.83 \times 10^{-4}$ & $7.07 \times 10^{-4}$ & $4.00 \times 10^{-4}$ & $7.45 \times 10^{-4}$ \\
\hline Coulomb + viscous model & $1.03 \times 10^{-4}$ & $4.56 \times 10^{-4}$ & $1.29 \times 10^{-4}$ & $6.68 \times 10^{-4}$ \\
\hline Nonlinear model & $8.88 \times 10^{-5}$ & $2.71 \times 10^{-4}$ & $9.53 \times 10^{-5}$ & $4.49 \times 10^{-4}$ \\
\hline
\end{tabular}

Table 5. RSME of the trajectory tracking experiments

\section{Conclusions}

In order to realize the high-speed and high-accuracy motion control of parallel manipulator, nonlinear control method is used to improve the traditional dynamic controllers such as the APD controller and the CT controller. The common feature of the two controllers is eliminating tracking error by linear PD control, and the friction compensation is realized by using the Coulomb + viscous friction model. However, the linear PD control is not robust against the uncertain factors such as modeling error and external disturbance. To overcome this problem, the NPD control is combined with the conventional control strategies and two nonlinear dynamic controllers are developed. Moreover, a nonlinear model is used to construct the friction of the parallel manipulator, and the nonlinear friction can be compensated effectively. Our theory analysis implies that, the proposed controllers can guarantee asymptotic convergence to zero of both tracking error and error rate. And for its simple structures and design, the proposed controllers are easy to be realized for the industry applications of parallel manipulators. Our experiment results show that, the position error of the end-effector decrease obviously with the proposed controllers and the nonlinear friction compensation method, especially at the high-speed. So the nonlinear dynamic controller and nonlinear friction compensation can realize high-speed and highaccuracy trajectory tracking of the parallel manipulator in practice. Also these new methods can be used to other manipulators, such as serial ones, or parallel manipulator without redundant actuation to realize high-speed and high accuracy motion.

\section{Acknowledgments}

This work was supported by the National Natural Science Foundation of China with Grant No. 50905172, the Anhui Provincial Natural Science Foundation with Grant No.090412040, and the Fundamental Research Funds for the Central Universities.

\section{References}

Cheng H., Yiu Y.K., Li Z.X. (2003) Dynamics and control of redundantly actuated parallel manipulators. IEEE Trans. Mechatronics, 8(4): 483-491 
Ghorbel F.H., Chetelat O., Gunawardana R., Longchamp R. (2000) Modeling and set point control of closed-chain mechanisms: theory and experiment. IEEE Trans. Control Syst. Tech., 8(5):801-815

Hensen R.H.A., Angelis G.Z., Molengraft M.J.G., Jager A.G., Kok J.J. (2000) Grey-box modeling of friction: An experimental case-study. European Journal of Control 6(3):258-267

Han J.Q. (1994) Nonlinear PID controller. Acta Automatica Sinica, 20(4): 487-490.

Kelly R., Ricardo C. (1996) A class of nonlinear PD-type controller for robot manipulator. Journal of Robotic Systems, 13: 793-802

Kostic D., Jager B., Steinbuch M., Hensen R. (2004) Modeling and identification for highperformance robot control: an RRR-robotic arm case study. IEEE Trans. Contr. Syst. Techn. 12(6): 904-919

Li Q., Wu F.X. (2004) Control performance improvement of a parallel robot via the design for control approach. Mechatronics, 14(8): 947-964

Merlet J.P. (2000) Parallel robots. Norwell, MA: Kluwer

Muller A. (2005) Internal preload control of redundantly actuated parallel manipulators - Its application to backlash avoiding control, IEEE Trans. Robotics, 21(4), 668 - 677

Murray R., Li Z. X., Sastry S. (1994) A Mathematical Introduction to Robotic Manipulation. CRC Press

Ouyang P.R., Zhang W.J., Wu F.X. (2002) Nonlinear PD control for trajectory tracking with consideration of the design for control methodology. In: Proc. of the IEEE Int. Conf. Robot. Autom., Washington; May, 2002, pp. 4126-4131

Paccot F., Andreff N., Martinet P. (2009) A review on the dynamic control of parallel kinematic machines: theory and experiments. International Journal of Robotics Research, 28(3): 395-416

Seraji H. (1998) A new class of nonlinear PID controllers with robotic applications. Journal of Robotic Systems, 15(3): 161-181

Shang W.W., Cong S., Zhang Y.X. (2008) Nonlinear friction compensation of a 2-DOF planar parallel manipulator. Mechatronics, 18(7): 340-346

Shang W. W., Cong S. (2009) Nonlinear computed torque control for a high speed planar parallel manipulator, Mechatronics, 19(6): 987-992

Shang W. W., Cong S., Li Z. X., Jiang S. L. (2009) Augmented nonlinear PD controller for a redundantly actuated parallel manipulator. Advanced Robotics, 23: (12-13), 1725-1742

Shang W. W., Cong S., Kong F. R. (2010) Identification of dynamic and friction parameters of a parallel manipulator with actuation redundancy, Mechatronics, 20(2): 192-200.

Shang W. W., Cong S., Jiang S. L. (2010) Dynamic model based nonlinear tracking control of a planar parallel manipulator, Nonlinear Dynamics, 60(4): 597-606

Slotine J. -J. E., Li W. P. (1991) Applied nonlinear control, Englewood Cliffs, N. J.: Prentice Hall.

Su Y.X., Duan B.Y., Zheng C.H., Zhang Y.F. Chen G.D., Mi J.W. (2004) Disturbance-rejection high-precision motion control of a Stewart platform. IEEE Trans. Control Syst. Tech., 12(3): 364-374

Su Y.X., Zheng C.H., Duan B.Y. (2005) Fuzzy learning tracking of a parallel cable manipulator for the square kilometer array. Mechatronics, 15(6):731-746

Wu F.X., Zhang W.J., Li Q., Ouyang P.R. (2002) Integrated design and PD control of highspeed closed-loop mechanisms. J. Dyn. Syst., Meas., Control, 124(4): 522-528

Xu Y., Hollerbach J.M., Ma D. (1995) A nonlinear PD controller for force and contact transient control. IEEE Control Systems Magazine, 15: 15-21.

Zhang Y.X., Cong S., Shang W.W., Li Z.X., and Jiang S.L. (2007) Modeling, identification and control of a redundant planar 2-Dof parallel manipulator. Int. J. Control, Autom. Syst., 5(5):559-569 


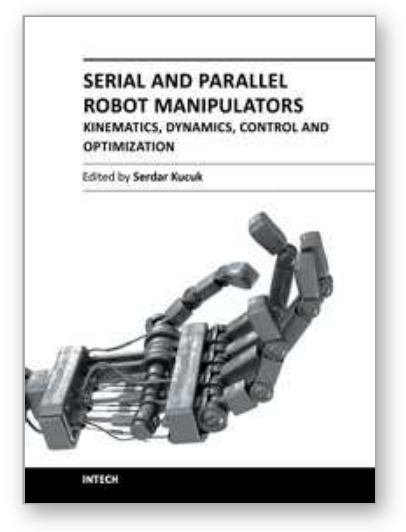

\section{Serial and Parallel Robot Manipulators - Kinematics, Dynamics, Control and Optimization}

Edited by Dr. Serdar Kucuk

ISBN 978-953-51-0437-7

Hard cover, 458 pages

Publisher InTech

Published online 30, March, 2012

Published in print edition March, 2012

The robotics is an important part of modern engineering and is related to a group of branches such as electric \& electronics, computer, mathematics and mechanism design. The interest in robotics has been steadily increasing during the last decades. This concern has directly impacted the development of the novel theoretical research areas and products. This new book provides information about fundamental topics of serial and parallel manipulators such as kinematics \& dynamics modeling, optimization, control algorithms and design strategies. I would like to thank all authors who have contributed the book chapters with their valuable novel ideas and current developments.

\section{How to reference}

In order to correctly reference this scholarly work, feel free to copy and paste the following:

Weiwei Shang and Shuang Cong (2012). Nonlinear Dynamic Control and Friction Compensation of Parallel Manipulators, Serial and Parallel Robot Manipulators - Kinematics, Dynamics, Control and Optimization, Dr. Serdar Kucuk (Ed.), ISBN: 978-953-51-0437-7, InTech, Available from:

http://www.intechopen.com/books/serial-and-parallel-robot-manipulators-kinematics-dynamics-control-andoptimization/nonlinear-dynamic-control-and-friction-compensation-of-parallel-manipulators

\section{INTECH}

open science | open minds

\section{InTech Europe}

University Campus STeP Ri

Slavka Krautzeka 83/A

51000 Rijeka, Croatia

Phone: +385 (51) 770447

Fax: +385 (51) 686166

www.intechopen.com

\section{InTech China}

Unit 405, Office Block, Hotel Equatorial Shanghai

No.65, Yan An Road (West), Shanghai, 200040, China

中国上海市延安西路65号上海国际贵都大饭店办公楼 405 单元

Phone: +86-21-62489820

Fax: +86-21-62489821 
(C) 2012 The Author(s). Licensee IntechOpen. This is an open access article distributed under the terms of the Creative Commons Attribution 3.0 License, which permits unrestricted use, distribution, and reproduction in any medium, provided the original work is properly cited. 